\title{
An Unfitted Discontinuous Galerkin Method for Elliptic Interface Problems
}

\author{
Qiuliang Wang ${ }^{1,2}$ and Jinru Chen ${ }^{1}$ \\ ${ }^{1}$ Jiangsu Key Laboratory for NSLSCS, School of Mathematical Sciences, Nanjing Normal University, Nanjing 210023, China \\ ${ }^{2}$ School of Mathematics and Information Science, Shangqiu Normal University, Shangqiu 476000, China \\ Correspondence should be addressed to Jinru Chen; jrchen@njnu.edu.cn
}

Received 7 November 2013; Accepted 1 January 2014; Published 20 February 2014

Academic Editor: Vit Dolejsi

Copyright (c) 2014 Q. Wang and J. Chen. This is an open access article distributed under the Creative Commons Attribution License, which permits unrestricted use, distribution, and reproduction in any medium, provided the original work is properly cited.

\begin{abstract}
An unfitted discontinuous Galerkin method is proposed for the elliptic interface problems. Based on a variant of the local discontinuous Galerkin method, we obtain the optimal convergence for the exact solution $u$ in the energy norm and its flux $\mathbf{p}$ in the $L^{2}$ norm. These results are the same as those in the case of elliptic problems without interface. Finally, some numerical experiments are presented to verify our theoretical results.
\end{abstract}

\section{Introduction}

Elliptic interface problems are often encountered in many multiphysics and multiphase applications in science computing and engineering. For example, second order elliptic equations with discontinuous coefficients are often used to model problems in material sciences and fluid dynamics when two or more distinct materials or fluids with different conductivities, densities, or permeability are involved. It is well known that, when the interface is smooth enough, the solution of elliptic interface problems has higher regularity in individual material or fluid region than in the entire physical domain.

To numerically solve such interface problems, first we need to generate a mesh. One approach is to use a body fitted mesh. However, for those problems where the interface moves with time, repeated remeshing of the domain to obtain a fitted mesh is very costly. Another one is to use an unfitted grid independent of the location of the interface. This technique is particularly preferred to simulate time-dependent problems with moving interfaces. The major advantage for using an unfitted mesh is that it avoids repeatedly remeshing the domain for fitting the moving interfaces.

As for fitted mesh method for elliptic problems with interface, Chen and Zou in [1] considered the finite element method for solving elliptic and parabolic interface problems, and almost-optimal error estimates in the $L^{2}$ norm and energy norm were obtained. In [2], the authors studied a class of discontinuous Galerkin method for elliptic interface problems, which was shown to be optimally convergent in $L^{2}$ norm. Recently, a high-order HDG method was presented to solve elliptic interface problems by Huynh et al. in [3], which was extended to solve Stokes interface flow in [4].

Various unfitted grid methods for interface problems have been proposed in the literature. Finite difference methods are very popular unfitted grid methods due to their simplicity, for example, the immersed interface method $[5,6]$, the immersed boundary method [7], the boundary condition capturing method [8], and many others. And there exist many works for finite element methods on unfitted grid as well. Babuška in [9] studied the elliptic interface problem on unfitted mesh and derived suboptimal convergence behavior. $\mathrm{Li}$ et al. proposed an immersed interface finite element method in [10], which modified the basis functions near interface to satisfy the homogeneous jump conditions. Later, this method was applied to elliptic and elasticity interface problems with nonhomogeneous jump conditions in $[11,12]$. Recently, an unfitted finite element method based on Nitsche's method was presented by A. Hansbo and P. Hansbo in [13] and optimal order of convergence was proved without restrictions on the location of the interface relative to the mesh, which was used to solve incompressible elasticity with discontinuous modulus in [14]. More recently, Massjung in [15] considered an $h p$ unfitted discontinuous Galerkin 
method which was viewed as a generalization of Hansbo's method in [13]. An optimal convergence rate with respect to $h$ and a suboptimal convergence rate with respect to $p$ in energy norm were proved. Later, $\mathrm{Wu}$ and Xiao also presented an unfitted $h p$ interface penalty finite element method, which was extended to the three dimensional case in [16].

The local discontinuous Galerkin (LDG) method was proposed by Cockburn and Shu in [17] to solve general timedependent convection-diffusion problems. Later, the method was carried to elliptic problems for mixed discontinuous Galerkin formulation by Castillo et al. in [18]. The purpose of this paper is to extend the LDG method to a class of elliptic problems with a smooth interface. However, employing an unfitted mesh method, the interface can divide regular grid cells into degenerated subcells. If this situation happens, the standard inverse estimates can no longer be valid. In this paper, we use the weighted average instead of the arithmetic average in the classic LDG method to retrieve the inverse estimates (see Lemmas 8 and 10). Thus, we propose an unfitted discontinuous Galerkin method, based on a variant of LDG method. We prove the optimal convergence rate of the method for the exact solution $u$ in the energy norm and its flux $\mathbf{p}$ in the $L^{2}$ norm, respectively.

The rest of this paper is organized as follows. In Section 2 we propose our DG method and present some necessary preliminaries. We prove optimal order error estimates for our DG method in Section 3. In Section 4, some numerical experiments are presented to justify our theoretical results. Finally, conclusions are given in Section 5.

Let us now end this section with some notation to be used in this paper. We will use the standard notations for Sobolev spaces and norms in this paper (see $[19,20])$. In particular, for a bounded open set $\Omega$ in $\mathbb{R}^{2}$, if $\Omega=\bigcup_{i=1}^{m} \Omega_{i}$ and $\Omega_{i} \cap \Omega_{j}=$ $\emptyset(i \neq j)$, we denote by $H^{k}\left(\bigcup_{i=1}^{m} \Omega_{i}\right)$ the Sobolev space of functions $u$ such that $\left.u\right|_{\Omega_{i}} \in H^{k}\left(\Omega_{i}\right)$, where $H^{k}\left(\Omega_{i}\right)$ denotes the standard Sobolev space with norm $\|\cdot\|_{k, \Omega_{i}}$. As usual we define the broken norm: $\|\cdot\|_{k, \Omega}=\left(\sum_{i=1}^{m}\|\cdot\|_{k, \Omega_{i}}^{2}\right)^{1 / 2}$. Throughout the paper, the generic constant $C$ is always independent of the mesh parameter $h$.

\section{Discontinuous Galerkin Method and Preliminaries}

Let $\Omega$ be a bounded domain in $\mathbb{R}^{2}$ with convex polygonal boundary $\partial \Omega$ and $\Omega^{+} \subset \Omega$ an open domain with $C^{2}$ boundary $\Gamma=\partial \Omega^{+} \subset \Omega$. Let $\Omega^{-}=\Omega \backslash \Omega^{+}$(see Figure 1). We consider the following elliptic interface problem:

$$
\begin{gathered}
-\nabla \cdot(\beta \nabla u)=f \text { in } \Omega^{+} \cup \Omega^{-}, \\
u=0 \text { on } \partial \Omega, \\
{[u]=0 \text { on } \Gamma,} \\
{\left[\beta \frac{\partial u}{\partial \mathbf{n}}\right]=g \text { on } \Gamma,}
\end{gathered}
$$

where $\mathbf{n}$ is the outward pointing unit normal to $\Omega^{+}$and $[u]:=\left.u^{+}\right|_{\Gamma}-\left.u^{-}\right|_{\Gamma}$ is the jump of $u$ across the interface $\Gamma$, where $u^{ \pm}$is the restrictions of $u$ on $\Omega^{ \pm}$. For the sake of

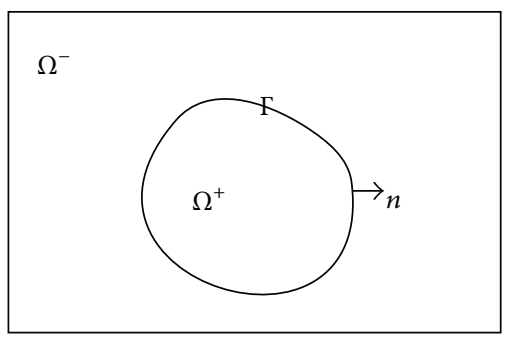

Figure 1: Domain $\Omega$, its subdomains $\Omega^{+}, \Omega^{-}$, and interface $\Gamma$.

simplicity, we assume that the coefficient $\beta$ is a positive and piecewise constant; that is, $\left.\beta\right|_{\Omega^{ \pm}}=\beta^{ \pm}>0$.

Regarding the regularity for the solution of the interface problem (1), we state without proof the following theorem.

Theorem 1 (cf. [1]). Assume that $f \in L^{2}(\Omega)$ and $g \in H^{1 / 2}(\Gamma)$. Then problem (1) has a unique solution $u \in H^{2}\left(\Omega^{+} \cup \Omega^{-}\right)$, and the following a priori estimate holds:

$$
\|u\|_{2, \Omega^{+} \cup \Omega^{-}} \leq C\left(\|f\|_{0, \Omega}+\|g\|_{1 / 2, \Gamma}\right) .
$$

By introducing the flux $\mathbf{p}=\beta \nabla u$, the interface problem (1) can be rewritten into a first order system as

$$
\begin{gathered}
\frac{1}{\beta} \mathbf{p}=\nabla u \quad \text { in } \Omega^{+} \cup \Omega^{-}, \\
-\nabla \cdot \mathbf{p}=f \quad \text { in } \Omega^{+} \cup \Omega^{-}, \\
{[u]=0 \quad \text { on } \Gamma,} \\
{[\mathbf{p} \cdot \mathbf{n}]=g \quad \text { on } \Gamma,} \\
u=0 \quad \text { on } \partial \Omega .
\end{gathered}
$$

Let $\mathscr{T}_{h}$ be a shape regular and locally quasi-uniform simplicial triangulation of $\Omega$, generated independently of the location of the interface $\Gamma$. For the definition of shape regular and locally quasi-uniform, we refer to [20, 21]. Suppose $\mathscr{T}_{h}$ to be made of straight triangles $T$ with diameter $h_{T}$. As usual, let $h:=\max _{T \in \mathscr{T}_{h}} h_{T}$. The set of edges of the triangulation $\mathscr{T}_{h}$ is denoted by $\mathscr{E}_{h}$, and $\mathscr{E}_{h}^{o}$ is the set of interior edges of $\mathscr{T}_{h}$. For any element $T \in \mathscr{T}_{h}$, denote the part of $T$ in $\Omega^{ \pm}$by $T^{ \pm}$; that is, $T^{ \pm}=T \cap \Omega^{ \pm}$. For any edge $e \in \mathscr{C}_{h}$, let $e^{ \pm}=e \cap \Omega^{ \pm}$. We call the elements whose interiors are cut through by $\Gamma$ "interface elements", and denote the set of the interface elements by $\mathscr{T}_{h}^{o}$. For an interface element $T \in \mathscr{T}_{h}^{o}$, assume that $\Gamma_{T}$ is the part of interface $\Gamma$ intersecting $T$. For the geometrical features of the interface $\Gamma$, we give the following plausible assumptions (cf. $[13,15])$.

Assumption 2. We assume that $\Gamma$ intersects the boundary $\partial T$ of an element $T \in \mathscr{T}_{h}^{o}$ exactly twice and each (open) edge at most once.

Assumption 3. Let $\Gamma_{T, h}$ be the straight line segment connecting the points of intersection between $\Gamma$ and $\partial T$. We assume that $\Gamma_{T}$ is a function of length on $\Gamma_{T, h}$, in local coordinates:

$$
\begin{gathered}
\Gamma_{T, h}=\left\{(\xi, \eta): 0<\xi<\left|\Gamma_{T, h}\right|, \eta=0\right\}, \\
\Gamma_{T}=\left\{(\xi, \eta): 0<\xi<\left|\Gamma_{T, h}\right|, \eta=\delta(\xi)\right\} .
\end{gathered}
$$


Assumption 4. Suppose that $e=T_{1} \cap T_{2}$ with $T_{1}, T_{2} \in \mathscr{T}_{h}$; then there exist triangles $S_{1}^{ \pm} \subset T_{1}^{ \pm}$and $S_{2}^{ \pm} \subset T_{2}^{ \pm}$such that $e^{ \pm}=S_{1}^{ \pm} \cap S_{2}^{ \pm}$and

$$
\left|S_{1}^{ \pm}\right|+\left|S_{2}^{ \pm}\right| \geq C\left|e^{ \pm}\right|^{2}
$$

To formulate our numerical scheme, first we define two usual discontinuous finite element spaces as

$$
\begin{gathered}
\widetilde{V}_{h}=\left\{v_{h} \in L^{2}(\Omega):\left.v_{h}\right|_{T} \in P_{k}(T), \forall T \in \mathscr{T}_{h}\right\}, \\
\widetilde{\mathbf{W}}_{h}=\left\{\mathbf{q}_{h} \in\left(L^{2}(\Omega)\right)^{2}:\left.\mathbf{q}_{h}\right|_{T} \in P_{k}(T)^{2}, \forall T \in \mathscr{T}_{h}\right\},
\end{gathered}
$$

where $P_{k}(T)$ denotes the space of polynomials of degree less than or equal to $k(k \geq 1)$ on each element $T$.

We define our discontinuous finite element spaces as

$$
\begin{gathered}
V_{h}=\left\{v_{h}:\left.v_{h}\right|_{\Omega^{ \pm}}=v_{h}^{ \pm}, \text {where } v_{h}^{ \pm} \in \widetilde{V}_{h}\right\}, \\
\mathbf{W}_{h}=\left\{\mathbf{q}_{h}:\left.\mathbf{q}_{h}\right|_{\Omega^{ \pm}}=\mathbf{q}_{h}^{ \pm}, \text {where } \mathbf{q}_{h}^{ \pm} \in \widetilde{\mathbf{W}}_{h}\right\} .
\end{gathered}
$$

Following the notation of [22], let $e$ be an interior edge shared by two triangles $T_{1}$ and $T_{2}$ in $\mathscr{T}_{h}$. For a scalar valued function $v$, piecewise smooth on $\mathscr{T}_{h}$ with $v_{j}=\left.v\right|_{T_{j}}$, we define the jump and the weighted average of $v$ as

$$
\begin{gathered}
\llbracket v^{ \pm} \rrbracket=v_{1}^{ \pm} \mathbf{n}_{1}+v_{2}^{ \pm} \mathbf{n}_{2}, \quad\left\{\left\{v^{ \pm}\right\}\right\}_{w}=\mu_{1}^{ \pm} v_{1}^{ \pm}+\mu_{2}^{ \pm} v_{2}^{ \pm}, \\
\left\{\left\{v^{ \pm}\right\}\right\}^{w}=\mu_{2}^{ \pm} v_{1}^{ \pm}+\mu_{1}^{ \pm} v_{2}^{ \pm} \quad \text { on } e \subset \mathscr{E}_{h}^{o} .
\end{gathered}
$$

Similarly, for a vector valued function $\mathbf{w}$, piecewise smooth on $\mathscr{T}_{h}$ with $\mathbf{w}_{j}=\left.\mathbf{w}\right|_{T_{j}}$, we set

$$
\begin{aligned}
\llbracket \mathbf{w}^{ \pm} \rrbracket= & \mathbf{w}_{1}^{ \pm} \cdot \mathbf{n}_{1}+\mathbf{w}_{2}^{ \pm} \cdot \mathbf{n}_{2}, \quad\left\{\left\{\mathbf{w}^{ \pm}\right\}\right\}_{w}=\mu_{1}^{ \pm} \mathbf{w}_{1}^{ \pm}+\mu_{2}^{ \pm} \mathbf{w}_{2}^{ \pm}, \\
& \left\{\left\{\mathbf{w}^{ \pm}\right\}\right\}^{w}=\mu_{2}^{ \pm} \mathbf{w}_{1}^{ \pm}+\mu_{1}^{ \pm} \mathbf{w}_{2}^{ \pm} \quad \text { on } e \subset \mathscr{E}_{h}^{o},
\end{aligned}
$$

where $\mathbf{n}_{j}$ is the unit normal of $e$ pointing towards the outside of $T_{j}$ and $\mu_{j}^{ \pm}=\left|S_{j}^{ \pm}\right| /\left(\left|S_{1}^{ \pm}\right|+\left|S_{2}^{ \pm}\right|\right), j=1,2$. If $e$ is an edge on the boundary of $\Omega$, we define on $e$

$$
\llbracket v \rrbracket=v \mathbf{n}, \quad\{\{\mathbf{w}\}\}_{w}=\mathbf{w},
$$

where $\mathbf{n}$ denotes the unit outer normal of $e$ pointing towards the outside of $\Omega$.

For the weight average across interface $\Gamma$ of any piecewise smooth function $v$ discontinuous on $\Gamma$, we set

$$
\{v\}_{w}=\lambda^{+} v^{+}+\lambda^{-} v^{-}, \quad\{v\}^{w}=\lambda^{-} v^{+}+\lambda^{+} v^{-},
$$

where $\lambda^{+}+\lambda^{-}=1$ whose specific definitions will be given in Lemma 10.

For simplicity, for $T^{ \pm}=T \cap \Omega^{ \pm}$and $e^{ \pm}=e \cap \Omega^{ \pm}$, we define

$$
\begin{aligned}
& \langle v, w\rangle_{T}:=\left(v^{+}, w^{+}\right)_{T^{+}}+\left(v^{-}, w^{-}\right)_{T^{-}}, \\
& \langle v, w\rangle_{e}:=\left(v^{+}, w^{+}\right)_{e^{+}}+\left(v^{-}, w^{-}\right)_{e^{-}} .
\end{aligned}
$$

Following [18], testing the problem (3) by $\mathbf{q}_{h} \in \mathbf{W}_{h}$ and $v_{h} \in V_{h}$, respectively, using integration by parts and noting the identities $\llbracket \mathbf{q} v \rrbracket=\llbracket v \rrbracket\{\{\mathbf{q}\}\}_{w}+\{\{v\}\}^{w} \llbracket \mathbf{q} \rrbracket$ and $[v w]=$ $\{w\}_{w}[v]+\{v\}^{w}[w]$, we obtain our DG method: find $\left(\mathbf{q}_{h}, u_{h}\right) \in$ $\mathbf{W}_{h} \times V_{h}$, such that

$$
\begin{aligned}
& \sum_{T \in \mathscr{T}_{h}}\left\langle\frac{1}{\beta} \mathbf{p}_{h}, \mathbf{q}_{h}\right\rangle_{T}+\sum_{T \in \mathscr{T}_{h}}\left\langle u_{h}, \nabla \cdot \mathbf{q}_{h}\right\rangle_{T}-\sum_{e \in \mathscr{C}_{h}^{o}}\left\langle\left\{\left\{u_{h}\right\}\right\}^{w}, \llbracket \mathbf{q}_{h} \rrbracket\right\rangle_{e}-\sum_{T \in \mathscr{T}_{h}^{o}}\left(\left\{u_{h}\right\}^{w},\left[\mathbf{q}_{\mathbf{h}} \cdot \mathbf{n}\right]\right)_{\Gamma_{T}}=0 \\
& \sum_{T \in \mathscr{T}_{h}}\left\langle\mathbf{p}_{h}, \nabla v_{h}\right\rangle_{T}-\sum_{e \in \mathscr{C}_{h}}\left\langle\left\{\left\{\mathbf{p}_{h}\right\}\right\}_{w}, \llbracket v_{h} \rrbracket\right\rangle_{e} \\
& \quad-\sum_{T \in \mathscr{T}_{h}^{o}}\left(\left\{\mathbf{p}_{h} \cdot \mathbf{n}\right\}_{w},\left[v_{h}\right]\right)_{\Gamma_{T}} \\
& \quad+\sum_{e \in \mathscr{E}_{h}}\left\langle\frac{\gamma}{\left|e^{ \pm}\right|} \llbracket u_{h} \rrbracket, \llbracket v_{h} \rrbracket\right\rangle_{e}+\sum_{T \in \mathscr{T}_{h}^{o}} \frac{\gamma}{h_{T}}\left(\left[u_{h}\right],\left[v_{h}\right]\right)_{\Gamma_{T}} \\
&=\sum_{T \in \mathscr{T}_{h}}\left\langle f, v_{h}\right\rangle_{T}+\sum_{T \in \mathscr{T}_{h}^{o}}\left(g,\left\{v_{h}\right\}^{w}\right)_{\Gamma_{T}}
\end{aligned}
$$

for all $\left(\mathbf{q}_{h}, v_{h}\right) \in \mathbf{W}_{h} \times V_{h}$, where $\gamma>0$ is the stabilization parameter.

We define the bilinear and linear forms

$$
\begin{gathered}
a\left(\mathbf{p}_{h}, \mathbf{q}_{h}\right)=\sum_{T \in \mathscr{T}_{h}}\left\langle\frac{1}{\beta} \mathbf{p}_{h}, \mathbf{q}_{h}\right\rangle_{T}, \\
b\left(v_{h}, \mathbf{p}_{h}\right)=\sum_{T \in \mathscr{T}_{h}}\left\langle\mathbf{p}_{h}, \nabla v_{h}\right\rangle_{T}-\sum_{e \in \mathscr{C}_{h}}\left\langle\left\{\left\{\mathbf{p}_{h}\right\}\right\}_{w}, \llbracket v_{h} \rrbracket\right\rangle_{e} \\
\quad-\sum_{T \in \mathscr{T}_{h}^{o}}\left(\left\{\mathbf{p}_{h} \cdot \mathbf{n}_{w},\left[v_{h}\right]\right)_{\Gamma_{T}},\right. \\
c\left(u_{h}, v_{h}\right)=\sum_{e \in \mathscr{E}_{h}}\left\langle\frac{\gamma}{\left|e^{ \pm}\right|} \llbracket u_{h} \rrbracket, \llbracket v_{h} \rrbracket\right\rangle_{e} \\
+\sum_{T \in \mathscr{T}_{h}^{o}} \frac{\gamma}{h_{T}}\left(\left[u_{h}\right],\left[v_{h}\right]\right)_{\Gamma_{T}}, \\
F\left(v_{h}\right)=\sum_{T \in \mathscr{T}_{h}}\left\langle f, v_{h}\right\rangle_{T}+\sum_{T \in \mathscr{T}_{h}^{o}}\left(g,\left\{v_{h}\right\}^{w}\right)_{\Gamma_{T}} .
\end{gathered}
$$

And integral by parts yields that

$$
\begin{aligned}
b\left(u_{h}, \mathbf{q}_{h}\right)= & -\sum_{T \in \mathscr{T}_{h}}\left\langle u_{h}, \nabla \cdot \mathbf{q}_{h}\right\rangle_{T}+\sum_{e \in \mathscr{C}_{h}^{o}}\left\langle\left\{\left\{u_{h}\right\}\right\}^{w}, \llbracket \mathbf{q}_{h} \rrbracket\right\rangle_{e} \\
& +\sum_{T \in \mathscr{T}_{h}^{o}}\left(\left\{u_{h}\right\}^{w},\left[\mathbf{q}_{h} \cdot \mathbf{n}\right]\right)_{\Gamma_{T}}
\end{aligned}
$$

Hence, our DG approximation can be written as the following mixed variational problem: find $\left(\mathbf{q}_{h}, u_{h}\right) \in \mathbf{W}_{h} \times V_{h}$, such that

$$
\begin{gathered}
a\left(\mathbf{p}_{h}, \mathbf{q}_{h}\right)-b\left(u_{h}, \mathbf{q}_{h}\right)=0 \quad \forall \mathbf{q}_{h} \in \mathbf{W}_{h}, \\
b\left(\mathbf{p}_{h}, v_{h}\right)+c\left(u_{h}, v_{h}\right)=F\left(v_{h}\right) \quad \forall u_{h} \in V_{h} .
\end{gathered}
$$

For the exact $u$ of the interface problem (1) and $\mathbf{p}=\beta \nabla u$, using Theorem 1, we have $\llbracket u \rrbracket=0$, $\mathbf{p} \rrbracket=0$ on $e \in \mathscr{E}_{h}^{o}$, and $[u]=0,[\mathbf{p} \cdot \mathbf{n}]=g$ across $\Gamma$. Then the following consistency property holds:

$$
\begin{gathered}
a\left(\mathbf{p}, \mathbf{q}_{h}\right)-b\left(u, \mathbf{q}_{h}\right)=0, \\
b\left(\mathbf{p}, v_{h}\right)+c\left(u, v_{h}\right)=F\left(v_{h}\right) .
\end{gathered}
$$


Let the mesh-dependent norm $|\|\cdot\||_{h}$ be defined by

$$
\begin{aligned}
\left.\left\|v_{h}\right\|\right|_{h} ^{2}= & \sum_{T \in \mathscr{T}_{h}}\left\|\nabla v_{h}\right\|_{0, T^{ \pm}}^{2}+\sum_{e \in \mathscr{C}_{h}} \frac{1}{\left|e^{ \pm}\right|}\left\|\left[v_{h}\right]\right\|_{0, e^{ \pm}}^{2} \\
& +\sum_{T \in \mathscr{T}_{h}^{o}} \frac{1}{h_{T}}\left\|\left[v_{h}\right]\right\|_{0, \Gamma_{T}}^{2} .
\end{aligned}
$$

Theorem 5. Suppose that the stabilization parameter $\gamma$ is positive; then the DG method (16) defines a unique approximate solution $\left(\mathbf{p}_{h}, u_{h}\right) \in \mathbf{W}_{h} \times V_{h}$.

Proof. Since (16) is a square system, it is enough to show uniqueness. Let $f=0, g=0$. Setting $\mathbf{q}_{h}=\mathbf{p}_{h}$ and $v_{h}=u_{h}$, adding the two equations of (16), we have

$$
a\left(\mathbf{p}_{h}, \mathbf{p}_{h}\right)+c\left(u_{h}, u_{h}\right)=0
$$

which deduces $\mathbf{p}_{h} \equiv 0, \llbracket u_{h} \rrbracket=0$ on $\mathscr{E}_{h}$ and $\left[u_{h}\right]=0$ across $\Gamma$. As a consequence, the first equation of (16) becomes

$$
\sum_{T \in \mathscr{T}_{h}}\left\langle\nabla u_{h}, \mathbf{q}_{h}\right\rangle=0 \quad \forall \mathbf{q}_{h} \in \mathbf{W}_{h} .
$$

Hence, taking $\mathbf{q}_{h}=\nabla u_{h}$ implies $\nabla u_{h}=0$. Since $\llbracket u_{h} \rrbracket=0$ on $\mathscr{E}_{h}$ and $\left[u_{h}\right]=0$ across $\Gamma$, we conclude that $u_{h} \equiv 0$. This completes the proof.

The following lemma comes from the famous Stein's extension theorem.

Lemma 6 (cf. [19]). There exist two extension operators $E^{ \pm}$: $H^{k}\left(\Omega^{ \pm}\right) \rightarrow H^{k}(\Omega)$ for all nonnegative integers $k$ such that

$$
\left.\left(E^{ \pm} v\right)\right|_{\Omega^{ \pm}}=v, \quad\left\|E^{ \pm} v\right\|_{H^{k}(\Omega)} \leq C\|v\|_{H^{k}\left(\Omega^{ \pm}\right)}
$$

where $v \in H^{k}\left(\Omega^{ \pm}\right)$.

Next, we state a standard approximation lemma.

Lemma 7 (cf. $[20,21]$ ). Let $u \in H^{k+1}(T)$. Then for $m=$ 0,1 there exists a linear continuous operator $\Pi_{T}: H^{k+1}(T) \rightarrow$ $P_{k}(T)$ such that

$$
\begin{gathered}
\left\|u-\Pi_{T} u\right\|_{m, T} \leq C h_{T}^{k+1-m}\|u\|_{k+1, T}, \\
\left\|u-\Pi_{T} u\right\|_{L^{\infty}(T)} \leq C h_{T}^{k}\|u\|_{k+1, T}, \\
\left\|u-\Pi_{T} u\right\|_{0, \partial T} \leq C h_{T}^{k+1 / 2}\|u\|_{k+1, T} .
\end{gathered}
$$

The following two lemmas are variant inverse estimates involving interface $\Gamma$ which play important role in our analysis.

Lemma 8 (cf. [15]). Fore $\in \mathscr{E}_{h}$ let $e=T_{1} \cap T_{2}$; then for $\mathbf{q} \in \mathbf{W}_{h}$ the following inverse inequality holds:

$$
\left\|\{\{\mathbf{q}\}\}_{w}\right\|_{0, e^{ \pm}}^{2} \leq C\left|e^{ \pm}\right|^{-1} \sum_{i=1}^{2}\|\mathbf{q}\|_{0, T_{i}^{ \pm}}^{2} .
$$

Lemma 9 (cf. $[15,16])$. The following estimate holds for either $i=+$ or $i=-$, for any $v \in P_{k}(T)$ :

$$
\|v\|_{0, \Gamma_{T}} \leq C h_{T}^{-1 / 2}\|v\|_{0, T^{i}} .
$$

By Lemma 9, we can immediately obtain the following result.

Lemma 10. Let $T \in \mathscr{T}_{h}^{o}$; then there exists a positive constant $C$ such that

$$
\lambda^{ \pm}\|v\|_{0, \Gamma_{T}} \leq C h_{T}^{-1 / 2}\|v\|_{0, T^{ \pm}}, \quad \forall v \in P_{k}(T),
$$

where

$$
\lambda^{ \pm}= \begin{cases}\frac{1}{2} & \text { if (26) holds for both } T^{ \pm} \text {and } T^{\mp}, \\ 1 & \text { if (26) holds only for } T^{ \pm} \\ 0 & \text { if (26) holds only for } T^{\mp} .\end{cases}
$$

Lemma 11. Let $T \in \mathscr{T}_{h}^{o}$ and $v \in H^{1}(T)$; then we have

$$
\|v\|_{0, \Gamma_{T}} \leq C\left(h_{T}^{-1 / 2}\|v\|_{0, T}+h_{T}^{1 / 2}|v|_{1, T}\right) .
$$

Proof. Under Assumptions 2 and 3, the trace inequality (29) follows from Lemma 3 in [13] and a scaling argument.

\section{Error Estimate of Our DG Method}

Now, we define interpolation operator $\Pi$ by $\left.\Pi\right|_{T}:=\Pi_{T}$ and $\Pi:=(\Pi, \Pi)$. Let $\boldsymbol{\xi}_{\mathbf{p}}^{ \pm}=\mathbf{p}^{ \pm}-\Pi E^{ \pm} \mathbf{p}^{ \pm}, \boldsymbol{\eta}_{\mathbf{p}}^{ \pm}=\Pi E^{ \pm} \mathbf{p}^{ \pm}-\mathbf{p}_{h}^{ \pm}$and $\xi_{u}^{ \pm}=$ $u^{ \pm}-\Pi E^{ \pm} u^{ \pm}, \eta_{u}^{ \pm}=\Pi E^{ \pm} u^{ \pm}-u_{h}^{ \pm}$. To obtain the convergence result, we need to show the following approximate error estimates.

Lemma 12. Suppose that $u \in H^{k+1}\left(\Omega^{+} \cup \Omega^{-}\right)$and $\mathbf{p} \epsilon$ $\left(H^{k+1}\left(\Omega^{+} \cup \Omega^{-}\right)\right)^{2}$; then the following approximate estimates hold:

$$
\begin{aligned}
& \left.\sum_{T \in \mathscr{T}_{h}}\left\|\xi_{u}\right\|_{0, T^{ \pm}}^{2}+\sum_{e \in \mathscr{E}_{h}} \frac{1}{\left|e^{ \pm}\right|} \| \llbracket \xi_{u}\right] \|_{0, e^{ \pm}}^{2} \\
& +\sum_{T \in \mathscr{T}_{h}^{o}} \frac{1}{h_{T}}\left\|\left[\xi_{u}\right]\right\|_{0, \Gamma_{T}}^{2} \leq C h^{2 k}\|u\|_{k+1, \Omega^{+} \cup \Omega^{-}}^{2}, \\
& \sum_{T \in \mathscr{T}_{h}}\left\|\xi_{\mathbf{p}}\right\|_{0, T^{ \pm}}^{2}+\sum_{e \in \mathscr{E}_{h}}\left|e^{ \pm}\right|\left\|\left\{\left\{\boldsymbol{\xi}_{\mathbf{p}}\right\}\right\}_{w}\right\|_{0, e^{ \pm}}^{2} \\
& +\sum_{T \in \mathscr{T}_{h}^{o}} h_{T}\left\|\left\{\boldsymbol{\xi}_{\mathbf{p}} \cdot \mathbf{n}\right\}_{w}\right\|_{0, \Gamma_{T}}^{2} \leq C h^{2 k}\|\mathbf{p}\|_{k, \Omega^{+} \cup \Omega^{-}}^{2}
\end{aligned}
$$

Proof. We only need to show inequality (30); inequality (31) can be shown similarly. For the first term on the left-hand side of (30), by the inequality (22) from Lemma 7 we obtain

$$
\begin{aligned}
\left\|\xi_{u}\right\|_{0, T^{ \pm}}^{2} & =\left\|E^{ \pm} u^{ \pm}-\Pi E^{ \pm} u^{ \pm}\right\|_{0, T^{ \pm}}^{2} \\
& \leq\left\|E^{ \pm} u^{ \pm}-\Pi E^{ \pm} u^{ \pm}\right\|_{0, T}^{2} \leq C h^{2 k+2}\left\|E^{ \pm} u^{ \pm}\right\|_{k+1, T}^{2} .
\end{aligned}
$$

Summing over all triangles, it follows by Lemma 6 that

$$
\sum_{T \in \mathscr{T}_{h}}\left\|\xi_{u}\right\|_{0, T^{ \pm}}^{2} \leq C h^{2 k+2}\|u\|_{k+1, \Omega^{+} \cup \Omega^{-}}^{2}
$$


Next, we estimate the second term on the left-hand side of (30) as follows. Suppose that $e=T_{1} \cap T_{2}$ with $T_{1}, T_{2} \in \mathscr{T}_{h}$; using the inequality (23) from Lemma 7 yields

$$
\begin{aligned}
\frac{1}{\left|e^{ \pm}\right|}\left\|\llbracket \xi_{u} \rrbracket\right\|_{0, e^{ \pm}}^{2} & =\frac{1}{\left|e^{ \pm}\right|}\left\|\llbracket E^{ \pm} u^{ \pm}-\Pi E^{ \pm} u^{ \pm} \rrbracket\right\|_{0, e^{ \pm}}^{2} \\
& \leq\left\|\llbracket E^{ \pm} u^{ \pm}-\Pi E^{ \pm} u^{ \pm} \rrbracket\right\|_{L^{\infty}\left(e^{ \pm}\right)}^{2} \\
& \leq C h^{2 k}\left\|E^{ \pm} u^{ \pm}\right\|_{k+1, T_{1} \cup T_{2}}^{2} .
\end{aligned}
$$

Summing over all edges, using Lemma 6 implies that

$$
\sum_{e \in \mathscr{E}_{h}} \frac{1}{\left|e^{ \pm}\right|}\left\|\llbracket \xi_{u} \rrbracket\right\|_{0, e^{ \pm}}^{2} \leq C h^{2 k}\|u\|_{k+1, \Omega^{+} \cup \Omega^{-}}^{2} .
$$

Similarly, due to the inequality (24) from Lemma 7 and Lemmas 6 and 11, we find

$$
\sum_{T \in \mathcal{T}_{h}^{o}} \frac{1}{h_{T}}\left\|\left[\xi_{u}\right]\right\|_{0, \Gamma_{T}}^{2} \leq C h^{2 k}\|u\|_{k+1, \Omega^{+} \cup \Omega^{-}}^{2} .
$$

Thus the inequality (30) follows combining (33)-(36), which completes the proof.

Next we present a priori error estimate of the exact solution $u$ in the energy norm $|\|\cdot\||_{h}$ and its flux $\mathbf{p}$ in the $L^{2}$ norm.

Theorem 13. Let $(\mathbf{p}, u)$ be the solution of (3) and $\left(\mathbf{p}_{h}\right.$, $\left.u_{h}\right)$ the solution of $(16)$, respectively. Then, for $(\mathbf{p}, u) \in$ $\left(H^{k}\left(\Omega^{+} \cup \Omega^{-}\right)\right)^{2} \times H^{k+1}\left(\Omega^{+} \cup \Omega^{-}\right)$, the following error estimate holds:

$$
\begin{aligned}
\| \mathbf{p} & -\mathbf{p}_{h}\left\|_{0, \Omega^{+} \cup \Omega^{-}}+\left|\left\|u-u_{h}\right\|\right|_{h}\right. \\
& \leq C h^{k}\left(\|\mathbf{p}\|_{k, \Omega^{+} \cup \Omega^{-}}+\|u\|_{k+1, \Omega^{+} \cup \Omega^{-}}\right) .
\end{aligned}
$$

Proof. By using the consistency property (17), we have

$$
\begin{gathered}
a\left(\boldsymbol{\eta}_{\mathbf{p}}, \mathbf{q}_{h}\right)-b\left(\eta_{u}, \mathbf{q}_{h}\right)=a\left(\boldsymbol{\xi}_{\mathbf{p}}, \mathbf{q}_{h}\right)-b\left(\xi_{u}, \mathbf{q}_{h}\right) \\
b\left(\boldsymbol{\eta}_{\mathbf{p}}, v_{h}\right)+c\left(\eta_{u}, v_{h}\right)=b\left(\boldsymbol{\xi}_{\mathbf{p}}, v_{h}\right)+c\left(\xi_{u}, v_{h}\right) .
\end{gathered}
$$

Set $\mathbf{q}_{h}=\boldsymbol{\eta}_{\mathbf{p}}, v_{h}=\eta_{u}$ in (38) to give

$$
\begin{aligned}
& a\left(\boldsymbol{\eta}_{\mathbf{p}}, \boldsymbol{\eta}_{\mathbf{p}}\right)+c\left(\eta_{u}, \eta_{u}\right) \\
& \quad=a\left(\boldsymbol{\xi}_{\mathbf{p}}, \boldsymbol{\eta}_{\mathbf{p}}\right)-b\left(\xi_{u}, \boldsymbol{\eta}_{\mathbf{p}}\right)+b\left(\boldsymbol{\xi}_{\mathbf{p}}, \eta_{u}\right)+c\left(\xi_{u}, \eta_{u}\right) .
\end{aligned}
$$

Using (31) in Lemma 12 and $\varepsilon$-Cauchy-Schwartz inequality, we estimate the first term on the right-hand side of (39) as

$$
\begin{aligned}
a\left(\boldsymbol{\xi}_{\mathbf{p}}, \boldsymbol{\eta}_{\mathbf{p}}\right) & =\sum_{T \in \mathscr{T}_{h}}\left\langle\boldsymbol{\xi}_{\mathbf{p}}, \boldsymbol{\eta}_{\mathbf{p}}\right\rangle_{T}=\sum_{T \in \mathscr{T}_{h}}\left(\boldsymbol{\xi}_{\mathbf{p}}, \boldsymbol{\eta}_{\mathbf{p}}\right)_{T^{ \pm}} \\
& \leq\left(\sum_{T \in \mathscr{T}_{h}}\left\|\boldsymbol{\xi}_{\mathbf{p}}\right\|_{0, T^{ \pm}}^{2}\right)^{1 / 2}\left(\sum_{T \in \mathscr{T}_{h}}\left\|\boldsymbol{\eta}_{\mathbf{p}}\right\|_{0, T^{ \pm}}^{2}\right)^{1 / 2} \\
& \leq C h^{2 k}\|\mathbf{p}\|_{k, \Omega^{+} \cup \Omega^{-}}^{2}+\varepsilon \sum_{T \in \mathscr{T}_{h}}\left\|\boldsymbol{\eta}_{\mathbf{p}}\right\|_{0, T^{ \pm}}^{2} \cdot
\end{aligned}
$$

For the second term on the right-hand side of (39), by using (30) in Lemma 12 and trace inequalities from Lemmas 8 and 10 , we obtain

$$
\begin{aligned}
& b\left(\xi_{u}, \boldsymbol{\eta}_{\mathbf{p}}\right) \\
& =\sum_{T \in \mathscr{T}_{h}}\left\langle\boldsymbol{\eta}_{\mathbf{p}}, \nabla \xi_{u}\right\rangle_{T}-\sum_{e \in \mathscr{E}_{h}}\left\langle\left\{\left\{\boldsymbol{\eta}_{\mathbf{p}}\right\}\right\}_{w}, \llbracket \xi_{u} \rrbracket\right\rangle_{e} \\
& -\sum_{T \in \mathscr{T}_{h}^{o}}\left(\left\{\boldsymbol{\eta}_{\mathbf{p}} \cdot \mathbf{n}\right\}_{w},\left[\xi_{u}\right]\right)_{\Gamma_{T}} \\
& =\sum_{T \in \mathscr{T}_{h}}\left(\boldsymbol{\eta}_{\mathbf{p}}, \nabla \xi_{u}\right)_{T^{ \pm}}-\sum_{e \in \mathscr{C}_{h}}\left(\left\{\left\{\boldsymbol{\eta}_{\mathbf{p}}\right\}\right\}_{w}, \llbracket \xi_{u} \rrbracket\right)_{e^{ \pm}} \\
& -\sum_{T \in \mathscr{T}_{h}^{o}}\left(\left\{\boldsymbol{\eta}_{\mathbf{p}} \cdot \mathbf{n}\right\}_{w},\left[\xi_{u}\right]\right)_{\Gamma_{T}} \\
& \leq\left(\sum_{T \in \mathscr{T}_{h}}\left\|\boldsymbol{\eta}_{\mathbf{p}}\right\|_{0, T^{ \pm}}^{2}\right)^{1 / 2}\left(\sum_{T \in \mathscr{T}_{h}}\left\|\nabla \xi_{u}\right\|_{0, T^{ \pm}}^{2}\right)^{1 / 2} \\
& +\left(\sum_{e \in \mathscr{E}_{h}}\left|e^{ \pm}\right|\left\|\left\{\left\{\boldsymbol{\eta}_{\mathbf{p}}\right\}\right\}_{w}\right\|_{0, e^{ \pm}}^{2}\right)^{1 / 2} \\
& \times\left(\sum_{e \in \mathscr{E}_{h}} \frac{1}{\left|e^{ \pm}\right|}\left\|\llbracket \xi_{u} \rrbracket\right\|_{0, e^{ \pm}}^{2}\right)^{1 / 2} \\
& +\left(\sum_{T \in \mathscr{T}_{h}^{o}} h_{T}\left\|\left\{\boldsymbol{\eta}_{\mathbf{p}} \cdot \mathbf{n}\right\}_{w}\right\|_{0, \Gamma_{T}}^{2}\right)^{1 / 2}\left(\sum_{T \in \mathcal{T}_{h}^{o}} \frac{1}{h_{T}}\left\|\left[\xi_{u}\right]\right\|_{0, \Gamma_{T}}^{2}\right)^{1 / 2} \\
& \leq C h^{2 k}\|u\|_{k+1, \Omega^{+} \cup \Omega^{-}}^{2}+\varepsilon \sum_{T \in \mathscr{T}_{h}}\left\|\boldsymbol{\eta}_{\mathbf{p}}\right\|_{0, T^{ \pm}}^{2} .
\end{aligned}
$$

Similarly, by using the approximate estimation (31), the third term on (39) can be estimated as

$$
\begin{aligned}
& b\left(\xi_{\mathbf{p}}, \eta_{u}\right) \\
& =\sum_{T \in \mathscr{T}_{h}}\left\langle\boldsymbol{\xi}_{\mathbf{p}}, \nabla \eta_{u}\right\rangle_{T}-\sum_{e \in \mathscr{E}_{h}}\left\langle\left\{\left\{\boldsymbol{\xi}_{\mathbf{p}}\right\}\right\}_{w}, \llbracket \eta_{u} \rrbracket\right\rangle_{e} \\
& -\sum_{T \in \mathscr{T}_{h}^{o}}\left(\left\{\boldsymbol{\xi}_{\mathbf{p}} \cdot \mathbf{n}\right\}_{w},\left[\eta_{u}\right]\right)_{\Gamma_{T}} \\
& =\sum_{T \in \mathscr{T}_{h}}\left(\boldsymbol{\xi}_{\mathbf{p}}, \nabla \eta_{u}\right)_{T^{ \pm}}-\sum_{e \in \mathscr{E}_{h}}\left(\left\{\left\{\boldsymbol{\xi}_{\mathbf{p}}\right\}\right\}_{w}, \llbracket \eta_{u} \rrbracket\right)_{e^{ \pm}} \\
& -\sum_{T \in \mathscr{T}_{h}^{o}}\left(\left\{\boldsymbol{\xi}_{\mathbf{p}} \cdot \mathbf{n}\right\}_{w},\left[\eta_{u}\right]\right)_{\Gamma_{T}} \\
& \leq\left(\sum_{T \in \mathscr{T}_{h}}\left\|\boldsymbol{\xi}_{\mathbf{p}}\right\|_{0, T^{ \pm}}^{2}\right)^{1 / 2}\left(\sum_{T \in \mathscr{T}_{h}}\left\|\nabla \eta_{u}\right\|_{0, T^{ \pm}}^{2}\right)^{1 / 2} \\
& +\left(\sum_{e \in \mathscr{C}_{h}}\left|e^{ \pm}\right|\left\|\left\{\left\{\boldsymbol{\xi}_{\mathbf{p}}\right\}\right\}_{w}\right\|_{0, e^{ \pm}}^{2}\right)^{1 / 2} \\
& \times\left(\sum_{e \in \mathscr{E}_{h}} \frac{1}{\left|e^{ \pm}\right|}\left\|\llbracket \eta_{u} \rrbracket\right\|_{0, e^{ \pm}}^{2}\right)^{1 / 2} \\
& +\left(\sum_{T \in \mathscr{T}_{h}^{o}} h_{T}\left\|\left\{\boldsymbol{\xi}_{\mathbf{p}} \cdot \mathbf{n}\right\}_{w}\right\|_{0, \Gamma_{T}}^{2}\right)^{1 / 2}
\end{aligned}
$$




$$
\begin{aligned}
& \times\left(\sum_{T \in \mathscr{T}_{h}^{o}} \frac{1}{h_{T}}\left\|\left[\eta_{u}\right]\right\|_{0, \Gamma_{T}}^{2}\right)^{1 / 2} \\
\leq & C h^{2 k}\|\mathbf{p}\|_{k, \Omega^{+} \cup \Omega^{-}}^{2} \\
& +\varepsilon\left(\sum_{e \in \mathscr{E}_{h}} \frac{1}{\left|e^{ \pm}\right|_{0, e^{ \pm}}}\left\|\llbracket \eta_{u} \rrbracket\right\|_{0, e^{ \pm}}^{2}+\sum_{T \in \mathscr{T}_{h}} \frac{1}{h_{T}}\left\|\left[\eta_{u}\right]\right\|_{0, \Gamma_{T}}^{2}\right. \\
& \left.+\sum_{T \in \mathscr{T}_{h}}\left\|\nabla \eta_{u}\right\|_{0, T^{ \pm}}^{2}\right) .
\end{aligned}
$$

Then, by virtue of inequality (30) in Lemma 12, we bound the fourth term on (39) as

$$
\begin{aligned}
c\left(\xi_{u}, \eta_{u}\right)= & \sum_{e \in \mathscr{E}_{h}}\left\langle\frac{\gamma}{\left|e^{ \pm}\right|} \llbracket \xi_{u} \rrbracket, \llbracket \eta_{u} \rrbracket\right\rangle_{e}+\sum_{T \in \mathscr{T}_{h}^{o}} \frac{\gamma}{h_{T}}\left(\left[\xi_{u}\right],\left[\eta_{u}\right]\right)_{\Gamma_{T}} \\
= & \sum_{e \in \mathscr{E}_{h}}\left(\frac{\gamma}{\left|e^{ \pm}\right|} \llbracket \xi_{u} \rrbracket, \llbracket \eta_{u} \rrbracket\right)_{e^{ \pm}}+\sum_{T \in \mathscr{T}_{h}^{o}} \frac{\gamma}{h_{T}}\left(\left[\xi_{u}\right],\left[\eta_{u}\right]\right)_{\Gamma_{T}} \\
\leq & \left(\sum_{e \in \mathscr{E}_{h}} \frac{\gamma}{\left|e^{ \pm}\right|}\left\|\llbracket \llbracket \xi_{u} \rrbracket\right\|_{0, e^{ \pm}}^{2}\right)^{1 / 2} \\
& \times\left(\sum_{e \in \mathscr{E}_{h}} \frac{\gamma}{\left|e^{ \pm}\right|}\left\|\llbracket \eta_{u} \rrbracket\right\|_{0, e^{ \pm}}^{2}\right)^{1 / 2} \\
& +\left(\sum_{T \in \mathscr{T}_{h}^{o}} \frac{\gamma}{h_{T}}\left\|\left[\xi_{u}\right]\right\|_{0, \Gamma_{T}}^{2}\right)^{1 / 2} \\
& \times\left(\sum_{T \in \mathscr{T}_{h}^{o}} \frac{\gamma}{h_{T}}\left\|\left[\eta_{u}\right]\right\|_{0, \Gamma_{T}}^{2}\right)^{1 / 2} \\
\leq & C h^{2 k}\|u\|_{k+1, \Omega^{+} \cup \Omega^{-}}^{2}+\varepsilon c\left(\eta_{u}, \eta_{u}\right) .
\end{aligned}
$$

Thus, combining (39)-(43) yields

$$
\begin{aligned}
& a\left(\boldsymbol{\eta}_{\mathbf{p}}, \boldsymbol{\eta}_{\mathbf{p}}\right)+c\left(\eta_{u}, \eta_{u}\right) \\
& \leq C^{2 k}\left(\|\mathbf{p}\|_{k, \Omega^{+} \cup \Omega^{-}}^{2}+\|u\|_{k+1, \Omega^{+} \cup \Omega^{-}}^{2}\right) \\
& \quad+\varepsilon \sum_{T \in \mathscr{T}_{h}}\left\|\nabla \eta_{u}\right\|_{0, T^{ \pm}}^{2} .
\end{aligned}
$$

By using the triangle inequality, we have

$$
\begin{aligned}
\| \mathbf{p} & -\mathbf{p}_{h} \|_{0, \Omega^{+} \cup \Omega^{-}}^{2}+c\left(\eta_{u}, \eta_{u}\right) \\
& \leq C h^{2 k}\left(\|\mathbf{p}\|_{k, \Omega^{+} \cup \Omega^{-}}^{2}+\|u\|_{k+1, \Omega^{+} \cup \Omega^{-}}^{2}\right)+\varepsilon \sum_{T \in \mathscr{T}_{h}}\left\|\nabla \eta_{u}\right\|_{0, T^{+}}^{2} .
\end{aligned}
$$

At the other hand, setting $\mathbf{q}_{h}=\nabla \eta_{u}$ in the first equality of (38), we obtain

$$
a\left(\boldsymbol{\eta}_{\mathbf{p}}, \nabla \eta_{u}\right)-b\left(\eta_{u}, \nabla \eta_{u}\right)=a\left(\xi_{\mathbf{p}}, \nabla \eta_{u}\right)-b\left(\xi_{u}, \nabla \eta_{u}\right) .
$$

By the definition of $b(\cdot, \cdot)$, an integration by parts implies that

$$
\begin{aligned}
\sum_{T \in \mathscr{T}_{h}}\left\|\nabla \eta_{u}\right\|_{0, T^{ \pm}}^{2}= & a\left(\mathbf{p}-\mathbf{p}_{h}, \nabla \eta_{u}\right)+b\left(\xi_{u}, \nabla \eta_{u}\right) \\
& +\sum_{e \in \mathscr{C}_{h}}\left\langle\left\{\left\{\nabla \eta_{u}\right\}\right\}_{w}, \llbracket \eta_{u} \rrbracket\right\rangle_{e} \\
& +\sum_{T \in \mathscr{T}_{h}^{o}}\left(\left\{\nabla \eta_{u} \cdot \mathbf{n}\right\}_{w},\left[\eta_{u}\right]\right)_{\Gamma_{T}} .
\end{aligned}
$$

Using Lemmas 8 and 10 yields

$$
\begin{aligned}
\sum_{T \in \mathscr{T}_{h}}\left\|\nabla \eta_{u}\right\|_{0, T^{ \pm}}^{2} \leq & C\left\|\mathbf{p}-\mathbf{p}_{h}\right\|_{0, \Omega^{+} \Omega^{-}}^{2} \\
& +C h^{2 k}\|u\|_{k+1, \Omega^{+} \cup \Omega^{-}}+c\left(\eta_{u}, \eta_{u}\right) \\
& +\varepsilon \sum_{T \in \mathscr{T}_{h}}\left\|\nabla \eta_{u}\right\|_{0, T^{ \pm}}^{2} .
\end{aligned}
$$

Combining (45) and (48), choosing $\varepsilon$ enough small, from the definition $|\|\cdot\||_{h}$ and the triangle inequality, we can arrive at

$$
\begin{aligned}
\| \mathbf{p} & -\mathbf{p}_{h}\left\|_{0, \Omega^{+} \cup \Omega^{-}}^{2}+\mid\right\| u-u_{h} \|_{h}^{2} \\
& \leq C h^{2 k}\left(\|\mathbf{p}\|_{k, \Omega^{+} \cup \Omega^{-}}^{2}+\|u\|_{k+1, \Omega^{+} \cup \Omega^{-}}^{2}\right),
\end{aligned}
$$

which completes the proof.

Using the standard duality argument, we can obtain the following error estimate in the $L^{2}$ norm.

Theorem 14. Under the condition of Theorem 13, we have

$$
\left\|u-u_{h}\right\|_{0, \Omega^{+} \cup \Omega^{-}} \leq C h^{k+1}\left(\|\mathbf{p}\|_{k, \Omega^{+} \cup \Omega^{-}}+\|u\|_{k+1, \Omega^{+} \cup \Omega^{-}}\right) .
$$

Proof. Consider the following so-called adjoint problem

$$
\begin{gathered}
-\nabla \cdot(\beta \nabla \phi)=u-u_{h} \quad \text { in } \Omega_{1} \cup \Omega_{2}, \\
\phi=0 \quad \text { on } \partial \Omega, \\
{[\phi]=0 \quad \text { on } \Gamma,} \\
{\left[\beta \frac{\partial \phi}{\partial \mathbf{n}}\right]=0 \quad \text { on } \Gamma .}
\end{gathered}
$$

Using Theorem 1, we get

$$
\|\phi\|_{2, \Omega^{+} \cup \Omega^{-}} \leq C\left\|u-u_{h}\right\|_{0, \Omega^{+} \cup \Omega^{-}} .
$$

By introducing an auxiliary variable $\mathbf{q}=\beta \nabla \phi$, we obtain

$$
\begin{gathered}
\frac{1}{\beta} \mathbf{q}=\nabla \phi \quad \text { in } \Omega^{+} \cup \Omega^{-}, \\
-\nabla \cdot \mathbf{q}=u-u_{h} \quad \text { in } \Omega^{+} \cup \Omega^{-}, \\
\phi=0 \quad \text { on } \partial \Omega, \\
{[\phi]=0 \quad \text { on } \Gamma,} \\
{[\mathbf{q} \cdot \mathbf{n}]=0 \quad \text { on } \Gamma .}
\end{gathered}
$$


Since $[\phi]=0$ on $\Gamma$ and $\llbracket \phi \rrbracket=0$ on $\mathscr{E}_{h}$, using integration by parts and the consistency property (17), we deduce

$$
\begin{aligned}
\left\|u-u_{h}\right\|_{0, \Omega^{+} \cup \Omega^{-}}^{2}= & \left(u-u_{h}, u-u_{h}\right) \\
= & a\left(\mathbf{q}, \mathbf{p}-\mathbf{p}_{h}\right)-b\left(\phi, \mathbf{p}-\mathbf{p}_{h}\right) \\
& +b\left(u-u_{h}, \mathbf{q}\right)+c\left(\phi, u-u_{h}\right) \\
= & \sum_{T \in \mathscr{T}_{h}}\left\langle\frac{1}{\beta} \xi_{\mathbf{q}}, \mathbf{p}-\mathbf{p}_{h}\right\rangle_{T} \\
& -\sum_{T \in \mathscr{T}_{h}}\left\langle\nabla \xi_{\phi}, \mathbf{p}-\mathbf{p}_{h}\right\rangle_{T} \\
& +\sum_{e \in \mathscr{E}_{h}}\left\langle\left\{\left\{\mathbf{p}-\mathbf{p}_{h}\right\}\right\}_{w}, \llbracket \xi_{\phi} \rrbracket\right\rangle_{e} \\
& +\sum_{T \in \mathscr{T}_{h}^{o}}\left(\left\{\left(\mathbf{p}-\mathbf{p}_{h}\right) \cdot \mathbf{n}\right\}_{w},\left[\xi_{\phi}\right]\right)_{\Gamma_{T}} \\
& +\sum_{T \in \mathscr{T}_{h}}\left\langle\xi_{\mathbf{q}}, \nabla\left(u-u_{h}\right)\right\rangle_{T} \\
& -\sum_{e \in \mathscr{E}_{h}}\left\langle\left\{\left\{\xi_{\mathbf{q}}\right\}\right\}_{w}, \llbracket u-u_{h} \rrbracket\right\rangle_{e} \\
& -\sum_{T \in \mathscr{T}_{h}^{o}}\left(\left\{\xi_{\mathbf{q}} \cdot \mathbf{n}\right\}_{w},\left[u-u_{h}\right]\right)_{\Gamma_{T}} \\
& +\sum_{e \in \mathscr{E}_{h}}\left\langle\frac{1}{\left|e^{ \pm}\right|} \llbracket \xi_{\phi} \rrbracket, \llbracket u-u_{h} \rrbracket\right\rangle_{e} \\
& +\sum_{T \in \mathscr{T}_{h}^{\circ}} \frac{1}{h_{T}}\left(\left[\xi_{\phi}\right],\left[u-u_{h}\right]\right)_{\Gamma_{T}} \\
= & \sum_{i=1} E_{i} .
\end{aligned}
$$

Due to $\mathbf{q}=\beta \nabla \phi$, as in the proof of Theorem 13, an application of Theorem 1 implies that

$$
\begin{aligned}
& \left|E_{1}\right|+\left|E_{2}\right|+\sum_{i=5}^{9}\left|E_{i}\right| \\
& \quad \leq \operatorname{Ch}\|\phi\|_{2, \Omega^{+} \cup \Omega^{-}}\left(\left\|\mathbf{p}-\mathbf{p}_{h}\right\|_{0, \Omega^{+} \cup \Omega^{-}}+\mid\left\|u-u_{h}\right\| \|_{h}\right) .
\end{aligned}
$$

Now we estimate $E_{3}$ as follows:

$$
\begin{aligned}
E_{3}= & \sum_{e \in \mathscr{E}_{h}}\left\langle\left\{\left\{\boldsymbol{\xi}_{\mathbf{p}}\right\}\right\}_{w}, \llbracket \xi_{\phi} \rrbracket\right\rangle_{e}+\sum_{e \in \mathscr{E}_{h}}\left\langle\left\{\left\{\boldsymbol{\eta}_{\mathbf{p}}\right\}\right\}_{w^{\prime}} \llbracket \llbracket \xi_{\phi} \rrbracket\right\rangle_{e} \\
= & \left(\sum_{e \in \mathscr{E}_{h}}\left|e^{ \pm}\right|\left\|\left\{\left\{\boldsymbol{\xi}_{\mathbf{p}}\right\}\right\}_{w}\right\|_{0, e^{ \pm}}^{2}\right)^{1 / 2}\left(\sum_{e \in \mathscr{E}_{h}} \frac{1}{\left|e^{ \pm}\right|} \| \llbracket\left[\xi_{\phi} \rrbracket \|_{0, e^{ \pm}}^{2}\right)^{1 / 2}\right. \\
& +\left(\sum_{e \in \mathscr{E}_{h}}\left|e^{ \pm}\right|\left\|\left\{\left\{\boldsymbol{\eta}_{\mathbf{p}}\right\}\right\}_{w}\right\|_{0, e^{ \pm}}^{2}\right)^{1 / 2}\left(\sum_{e \in \mathscr{E}_{h}} \frac{1}{\left|e^{ \pm}\right|} \| \llbracket\left[\xi_{\phi} \rrbracket \|_{0, e^{ \pm}}^{2}\right)^{1 / 2}\right. \\
\leq & C h\left(h^{k}\|\mathbf{p}\|_{k, \Omega^{+} \cup \Omega^{-}}+\left\|\mathbf{p}-\mathbf{p}_{h}\right\|_{0, \Omega^{+} \cup \Omega^{-}}\right)\|\phi\|_{2, \Omega^{+} \cup \Omega^{-}}
\end{aligned}
$$

Similarly,

$$
\left|E_{4}\right| \leq C h\left(h^{k}\|\mathbf{p}\|_{k, \Omega^{+} \cup \Omega^{-}}+\left\|\mathbf{p}-\mathbf{p}_{h}\right\|_{0, \Omega^{+} \cup \Omega^{-}}\right)\|\phi\|_{2, \Omega^{+} \cup \Omega^{-}} .
$$

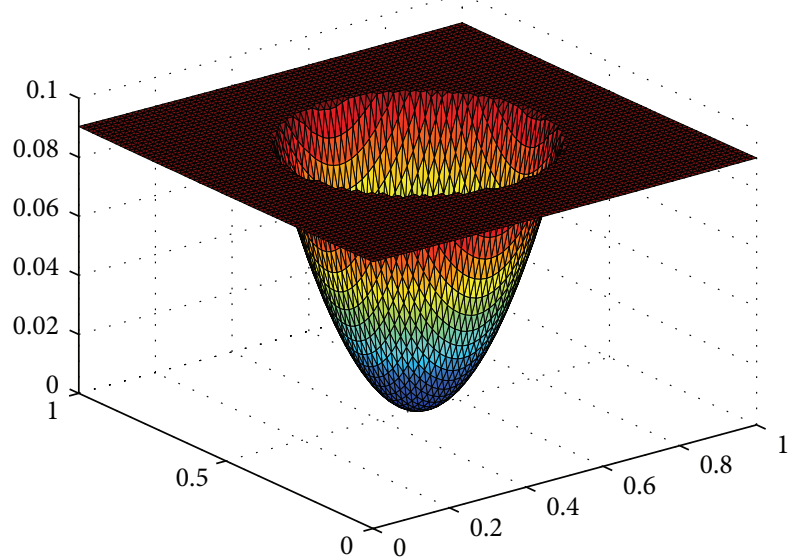

FIgURE 2: The numerical solution on a $64 \times 64$ uniform mesh for $\beta_{1}$ : $\beta_{2}=1: 1000$.

We now combine (54)-(57) and use (52) and Theorem 13 to obtain

$$
\left\|u-u_{h}\right\|_{0, \Omega^{+} \cup \Omega^{-}} \leq C h^{k+1}\left(\|\mathbf{p}\|_{k, \Omega^{+} \cup \Omega^{-}}+\|u\|_{k+1, \Omega^{+} \cup \Omega^{-}}\right),
$$

which completes the proof.

\section{Numerical Experiments}

In this section, we consider the following nontrivial example with a homogeneous jump condition (from $[10,13]$ ). The exact solution is given by

$$
u(x, y)= \begin{cases}\frac{r^{2}}{\beta_{1}} & \text { if } r \leq r_{0}, \\ \frac{r^{2}}{\beta_{2}}-\frac{r_{0}^{2}}{\beta_{2}}+\frac{r_{0}^{2}}{\beta_{1}}, & \text { if } r>r_{0},\end{cases}
$$

where $r=\sqrt{(x-0.5)^{2}+(y-0.5)^{2}}$, and on the domain $\Omega=$ $(0,1) \times(0,1)$ we choose $r_{0}=0.3$. A simple calculation shows that $\mathbf{p}(x, y)=(x, y)^{T}$.

We compute the order of convergence for $e_{u}=u-u_{h}$ and $\mathbf{e}_{\mathbf{p}}=\mathbf{p}-\mathbf{p}_{h}$, when piecewise linear polynomials are used to approximate $u$ and $\mathbf{p}$, respectively. In Figure 2, we plot the numerical solution for this example on a $64 \times 64$ uniform mesh for $\beta_{1}: \beta_{2}=1: 1000$. Figures $3,4,5$, and 6 show the computed order of convergence for $\left\|e_{u}\right\|_{0, \Omega_{1} \cup \Omega_{2}}$ and $\left\|\mathbf{e}_{\mathbf{p}}\right\|_{0, \Omega_{1} \cup \Omega_{2}}$ when the jump in the coefficient is taken as $\beta_{1}: \beta_{2}=1: 10,1: 1000,10: 1$, $1000: 1$, respectively, in the log-log scale. These computed results coincide with the theoretical results in Theorems 13 and 14.

\section{Conclusions}

In this paper, we have discussed an unfitted discontinuous Galerkin method for elliptic problems with a smooth interface. Based on a variant of local discontinuous Galerkin method, we have obtained the optimal order error estimates 


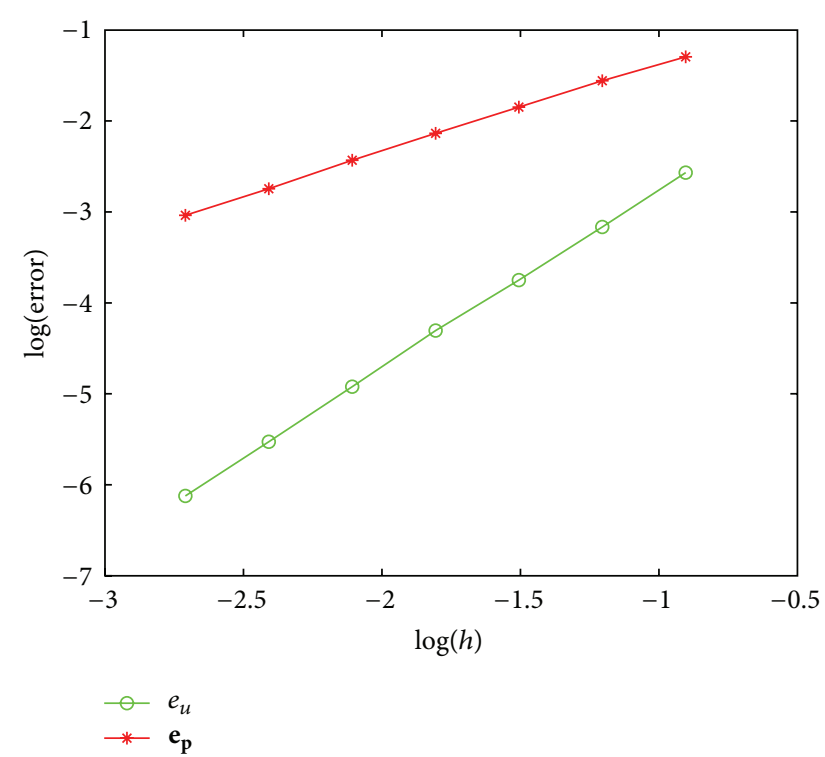

FIgURE 3: The convergence rates of $L^{2}$ norm in the exact $u$ and its flux $\mathbf{p}$ for $\beta_{1}: \beta_{2}=1: 10$.

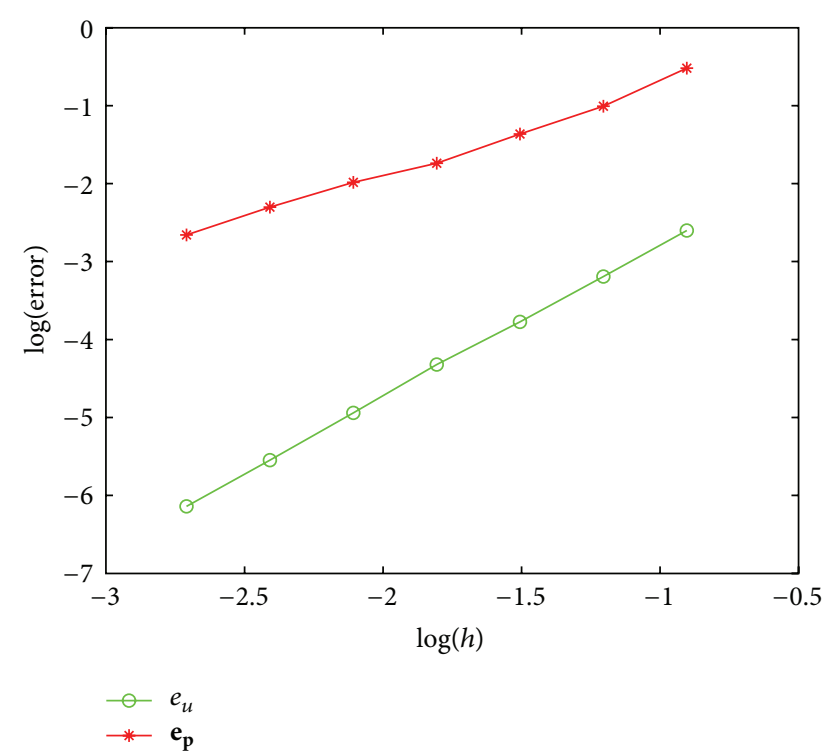

FIgURE 4: The convergence rates of $L^{2}$ norm in the exact $u$ and its flux $\mathbf{p}$ for $\beta_{1}: \beta_{2}=1: 1000$.

in the energy norm in $u$ and its flux p. And by using the standard duality argument the optimal convergence rate in $L^{2}$ norm for $u$ has also been derived. These presented results are the same as that of elliptic problems without interface. Finally, numerical experiments are given to confirm our theoretical results. We note that the convergence behavior in most existing works concerning the elliptic interface problems depends on the jump in the discontinuous coefficients. It will be one of our future subjects to design an efficient numerical scheme that is robust with respect to the jump in the discontinuous coefficients.

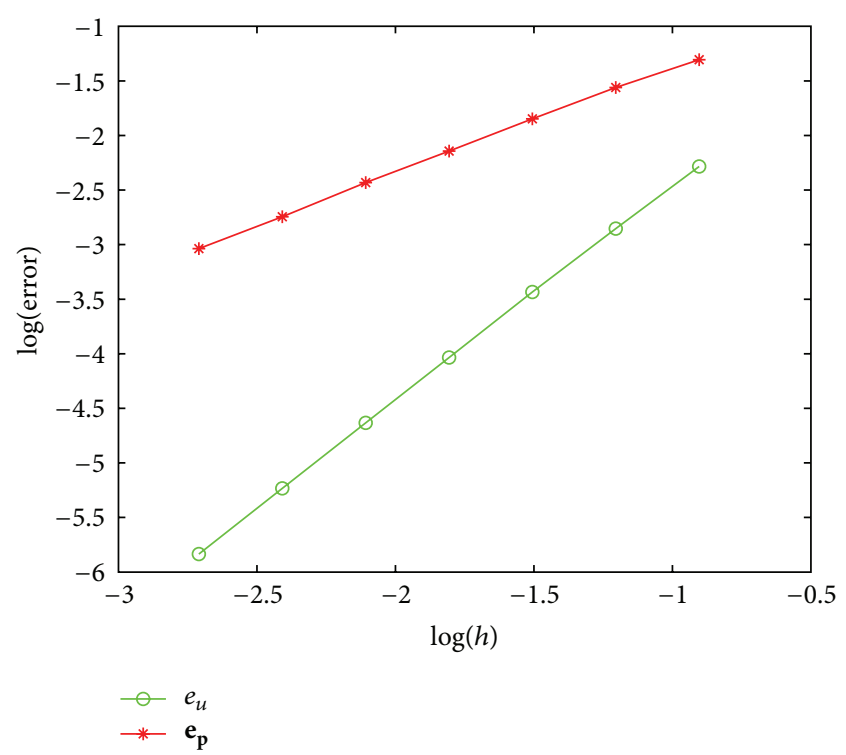

FIGURE 5: The convergence rates of $L^{2}$ norm in the exact $u$ and its flux $\mathbf{p}$ for $\beta_{1}: \beta_{2}=10: 1$.

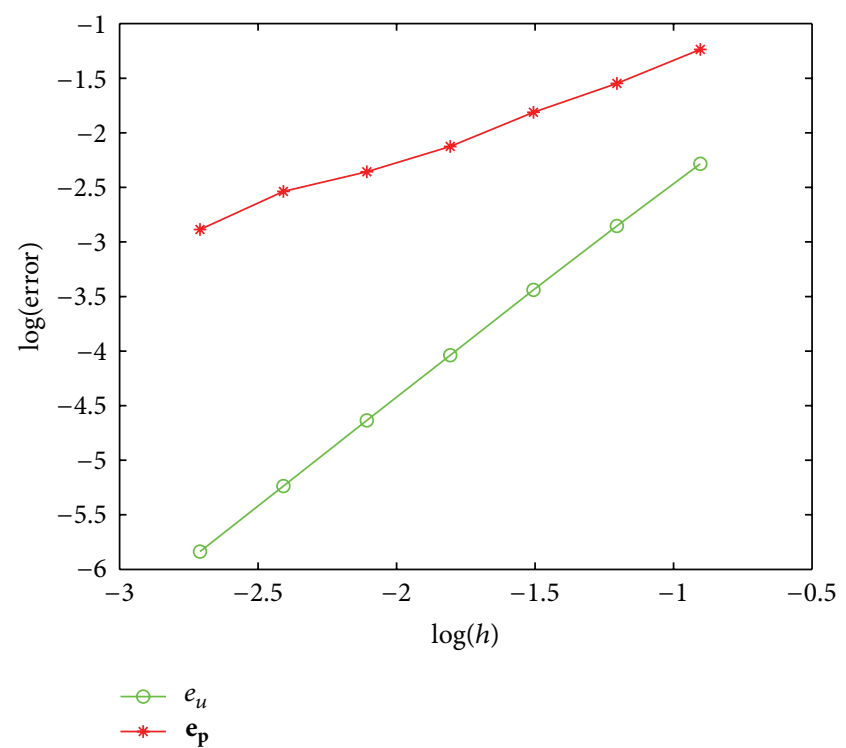

FIGURE 6: The convergence rates of $L^{2}$ norm in the exact $u$ and its flux $\mathbf{p}$ for $\beta_{1}: \beta_{2}=1000: 1$.

\section{Conflict of Interests}

The authors declare that there is no conflict of interests regarding the publication of this paper.

\section{Acknowledgments}

The authors would like to express sincere gratitude to the reviewers for their constructive suggestions which helped improve the quality of this paper. This work was supported by the National Science Foundation (NSF) of China (Grants no. 11371199 and 11301275), the Program of Natural Science 
Research of Jiangsu Higher Education Institutions of China (Grant no. 12KJB110013), and the Doctoral Fund of Ministry of Education of China (Grant no. 20123207120001).

\section{References}

[1] Z. Chen and J. Zou, "Finite element methods and their convergence for elliptic and parabolic interface problems," Numerische Mathematik, vol. 79, pp. 457-492, 1998.

[2] G. Guyomarc'h, C. Lee, and K. Jeon, “A discontinuous Galerkin method for elliptic interface problems with application to electroporation," Communications in Numerical Methods in Engineering, vol. 25, no. 10, pp. 991-1008, 2009.

[3] L. Huynh, N. Nguyen, J. Peraire, and B. Khoo, "A high order hybridizable discontinuous Galerkin method for elliptic interface problems," International Journal for Numerical Methods in Engineering, vol. 93, no. 2, pp. 183-200, 2013.

[4] B. Wang and B. Khoo, "Hybridizable discontinuous Galerkin method (HDG) for Stokes interface flow," Journal of Computational Physics, vol. 247, pp. 262-278, 2013.

[5] R. Leveque and Z. Li, "The immersed interface method for elliptic equations with discontinuous coefficients and singular sources," SIAM Journal on Numerical Analysis, vol. 31, no. 4, pp. 1019-1044, 1994.

[6] Z. Li and M. Lai, "The immersed interface method for the Navier-Stokes equations with singular forces," Journal of Computational Physics, vol. 171, no. 2, pp. 822-842, 2001.

[7] C. Peskin, "Numerical analysis of blood flow in the heart," Journal of Computational Physics, vol. 25, no. 3, pp. 220-252, 1977.

[8] X. Liu, R. Fedkiw, and M. Kang, "A boundary condition capturing method for Poisson's equation on irregular domains," Journal of Computational Physics, vol. 160, no. 1, pp. 151-178, 2000.

[9] I. Babuška, "The finite element method for elliptic equations with discontinuous coefficients," Computing, vol. 5, no. 3, pp. 207-213, 1970.

[10] Z. Li, T. Lin, and X. Wu, "New Cartesian grid methods for interface problems using the finite element formulation," Numerische Mathematik, vol. 96, no. 1, pp. 61-98, 2003.

[11] Y. Gong, B. Li, and Z. Li, "Immersed-interface finite-element methods for elliptic interface problems with non-homogeneous jump conditions," SIAM Journal on Numerical Analysis, vol. 35, no. 1, pp. 472-495, 2008.

[12] Y. Gong and Z. Li, "Immersed interface finite element methods for elasticity interface problems with non-homogeneous jump conditions," Numerical Mathematics: Theory, Methods and Applications, vol. 3, no. 1, pp. 23-39, 2010.

[13] A. Hansbo and P. Hansbo, "An unfitted finite element method, based on Nitsche's method, for elliptic interface problems," Computer Methods in Applied Mechanics and Engineering, vol. 191, pp. 5537-5552, 2002.

[14] R. Becker, E. Burman, and P. Hansbo, "A Nitsche extended finite element method for incompressible elasticity with discontinuous modulus of elasticity," Computer Methods in Applied Mechanics and Engineering, vol. 198, pp. 3352-3360, 2009.

[15] R. Massjung, "An unfitted discontinuous Galerkin method applied to elliptic interface problems," SIAM Journal on Numerical Analysis, vol. 50, no. 6, pp. 3134-3162, 2012.

[16] H. Wu and Y. Xiao, "An unfitted $h p$-interface penalty finite element method forelliptic interface problems," In press, http:// arxiv.org/abs/1007.2893.
[17] B. Cockburn and C. Shu, "The local discontinuous Galerkin method for time-dependent convection-diffusion systems," SIAM Journal on Numerical Analysis, vol. 35, no. 6, pp. 24402463, 1998.

[18] P. Castillo, B. Cockburn, I. Perugia, and D. Schötzau, "An a priori error analysis of the local discontinuous Galerkin method for elliptic problems," SIAM Journal on Numerical Analysis, vol. 38, no. 5, pp. 1676-1706, 2000.

[19] R. Adams and J. Fournier, Sobolev Spaces, Academic Press, New York, NY, USA, 2nd edition, 2003.

[20] P. Ciarlet, The Finite Element Method for Elliptic Problems, North-Holland, 1978.

[21] S. Brenner and L. Scott, The Mathematical Theory of Finite Element Methods, Springer, Berlin, Germany, 3rd edition, 2008.

[22] D. Arnold, F. Brezzi, B. Cockburn, and D. Marini, "Unified analysis of discontinuous Galerkin methods for elliptic problems," SIAM Journal on Numerical Analysis, vol. 39, no. 5, pp. 17491779,2002 


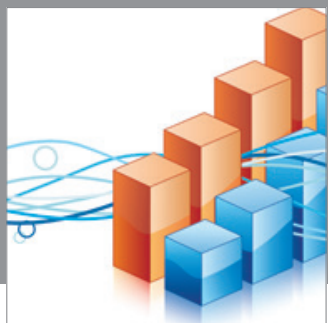

Advances in

Operations Research

mansans

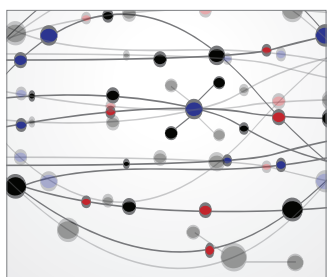

The Scientific World Journal
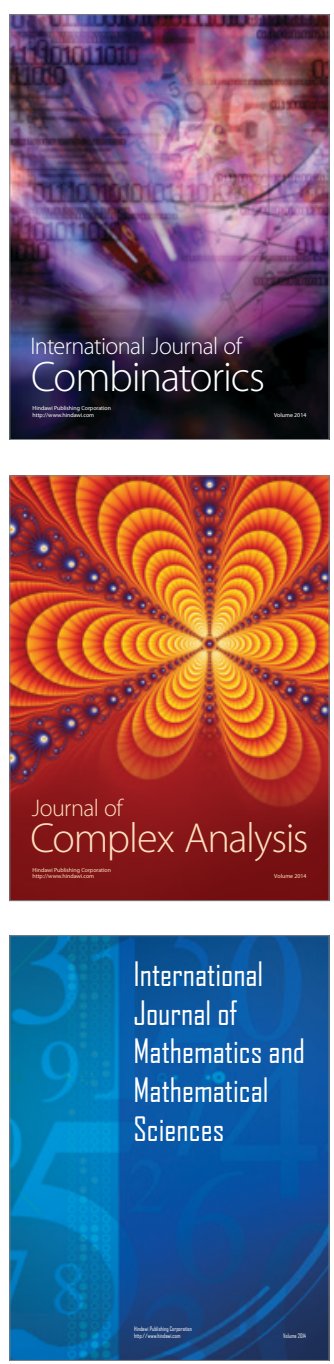
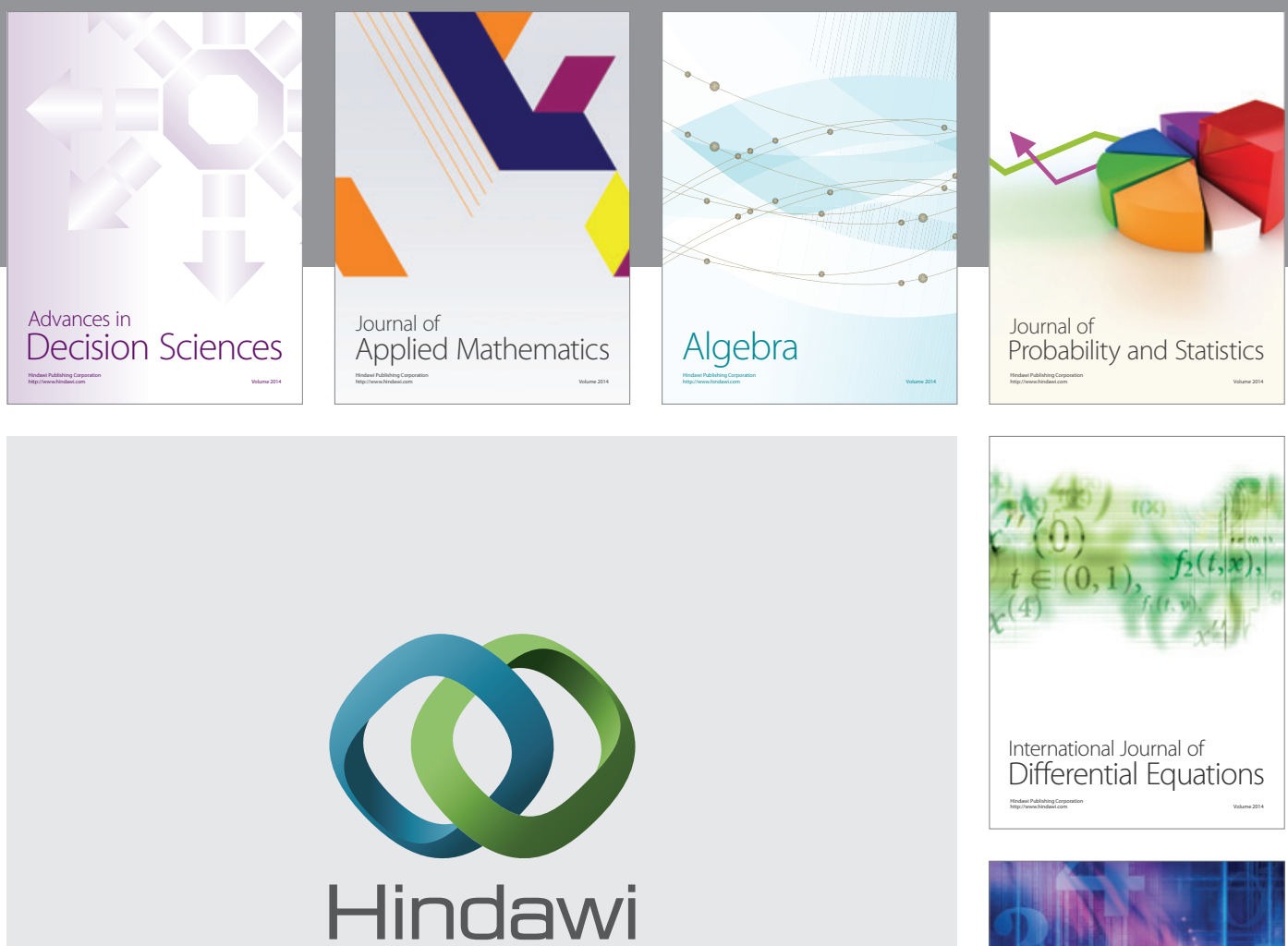

Submit your manuscripts at http://www.hindawi.com
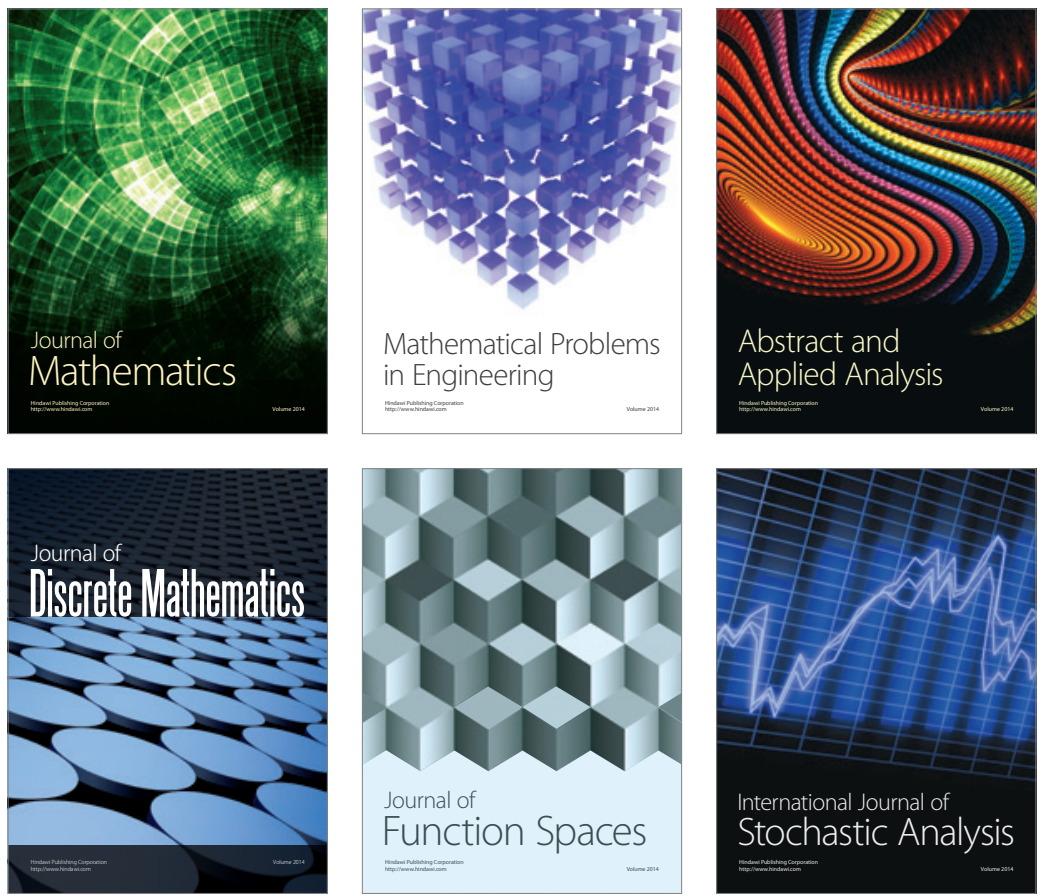

Journal of

Function Spaces

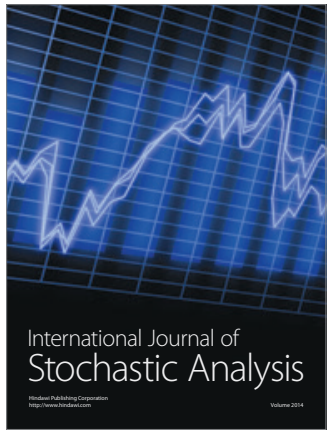

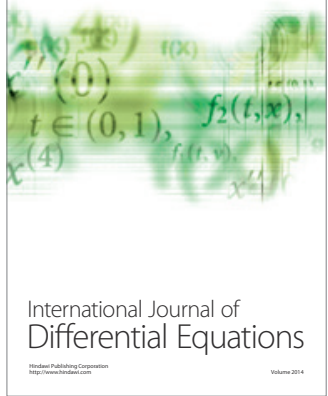
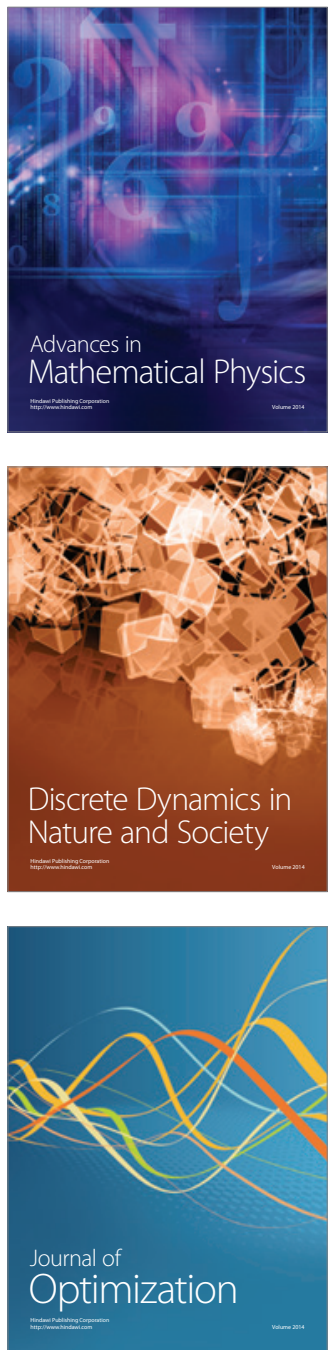